\title{
Hypercalcemia and Nephrogenic Diabetes Insipidus: Rare and Life-Threatening Effects of Lithium Intoxication
}

\begin{abstract}
Lithium is the most effective therapy for bipolar and schizoaffective disorders. Despite its efficacy, lithium has a narrow therapeutic index and adverse effects are frequent. Lithium intoxication (LI) generally affects brain, but less frequently can affect kidneys, thyroid, and parathyroid. Here, we report the case of a patient with lithium neurotoxic effects complicated by parathyroid and renal adverse effects. The patient was a 52-year-old woman treated with lithium, who was recently diagnosed with hypercalcemia and hyperparathyroidism.

She was admitted for severe agitation, confusion, and diffuse tremor. Despite serum lithium and calcium normalization, laboratory tests revealed a life-threatening hypernatremia caused by nephrogenic diabetes insipidus (NDI). Hemodialysis was started, but after the first treatment the patient died for cardiac arrest.

Neurological symptoms of LI may occur even if the dosage is close to the normal therapeutic range. Hypercalcemia and NDI are rare, but should be promptly diagnosed and treated. In case of poor clinical outcome, hemodialysis should be performed independently of lithium serum level.

Keywords: Lithium Toxicity; Hypercalcemia; Nephrogenic Diabetes Insipidus; Hypernatremia CMI 2020; 14(1): 45-49

http://dx.doi.org/10.7175/cmi.v14i1.1461
\end{abstract}

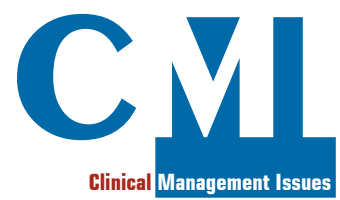

Case Report
Neurology Unit, Ospedale del Mare, ASL Napoli 1 Centro, Italy

2 Neurology and Stroke Unit, A.O.R.N. San G. Moscati, Avellino

\section{INTRODUCTION}

Lithium is the most effective long-term therapy for bipolar and schizoaffective disorder, protecting against both depression and mania and reducing the risk of suicide and short-term mortality. It is also used as add-on therapy in patients with treatmentresistant unipolar and recurrent major depression and in cluster headache prophylaxis [1]. Although its efficacy, lithium has a narrow therapeutic window and a clearance variability due to several individual and environmental factors. Therefore, lithium toxicity is frequent and routine monitoring of serum concentrations is required. Lithium intoxication (LI), whether intentional or unintentional, can be a severe occurrence carrying a significant risk for permanent sequelae, mainly neurologic, and can even lead to death. LI is categorized as:
- acute intoxication in lithium-naïve patients;

- acute-on-chronic intoxication in patients taking long-term lithium treatment; or

- chronic intoxication due to the gradual accumulation of lithium during chronic exposure [2].

\section{Why Do We Describe This Case}

Litbium is an effective medication but has a narrow therapeutic index and, thus, toxicity is frequent. Intoxication commonly involves central nervous system, but less frequently can cause rare and life-threatening adverse effects such as hypercalcemia and nephrogenic diabetes insipidus. These adverse effects should be promptly recognized and treated
Corresponding author Arturo de Falco arturodefalco@tin.it

Received: 7 March 2020 Accepted: 11 May 2020 Published: 30 September 2020 
The effective therapeutic range of lithium is $0.6-1.0 \mathrm{mmol} / \mathrm{L}$, but in long-term therapy levels equal or greater than $1.2 \mathrm{mmol} / \mathrm{L}$ are considered toxic. Several factors are associated with increased risk for LI:

- medications (i.e., neuroleptics, SSRIs, and amiodarone);

- dehydration;

- renal failure;

- infections;

- fever.

Further potential causes are intentional or accidental overdoses [3]. LI is associated with a variety of clinical manifestations depending on pathologic lithium accumulation in different organs including neurologic, gastrointestinal, endocrine, and renal disorders. Acute LI is most often associated with gastrointestinal symptoms, cardiotoxic effects, and late developing neurological signs, whereas chronic forms are predominantly characterized by neurological symptoms including tremor, extrapyramidal and/ or cerebellar signs, hyperreflexia, agitation, confusion, myoclonus, seizures, and consciousness impairment [3].

Lithium therapy is also associated with renal impairment [2,4,5]. Young women had higher hazard ratios than other groups, suggesting that they are at greatest risk of renal disorder [5]. A patient receiving lithium is at high risk of developing at least stage 3 chronic kidney disease. A subset of these patients might develop end-stage renal failure with an absolute risk of approximately 0.5 $2 \%[4,5]$. Increased serum lithium concentrations and young age in women seem to be associated with increased risk of decline in renal function [5].

Nephrogenic diabetes insipidus (NDI) is a rare adverse effect of LI: tubular cells lose their ability to respond to antidiuretic hormone (ADH). This effect is initially reversible, but can progress to structural and irreversible changes $[2,6]$. In this setting, the risk of hypernatremia increases and may lead to serious or even life-threatening conditions if not promptly and properly treated [7].

Antithyroid effects of lithium are well established and more evident in young women $[4,5]$. Multiple mechanisms are probably involved. The most important is the inhibition of thyroid hormone release from the thyroid gland. However, lithium may also decrease iodine trapping within the gland and inhibit thyroid hormones synthesis [8]. Although thyroid dysfunction is the most common endocrine adverse effect, patients receiving lithium have an absolute risk of $10 \%$ (vs. $0.1 \%$ of the general population) of developing primary hyperparathyroidism. This is probably caused by lithium inactivation of the calcium-sensing receptor leading to an increased release of parathyroid hormone, which raises calcium concentration in blood $[9,10]$. Moreover, in a review of 88 case reports of lithiuminduced hyperparathyroidism, 42 cases were found to have parathyroid adenomas and 29 were found to have hyperplasia $[9,10]$. Lithium treatment is also associated with cardiac conduction abnormalities and electrocardiographic changes such as transient ST depression, bradycardia, sinus node dysfunction, and inverted $\mathrm{T}$-waves in lateral precordial leads [2,11]. Lithium-treated patients who have hypercalcemia have been reported to have higher prevalence of cardiac conduction disturbances compared with lithium-treated patients with normal calcium levels $[9,11]$. The standard treatment strategy of lithium toxicity generally begins with cessation of lithium administration and medications that may reduce lithium elimination, hydration, and gastrointestinal decontamination. Because of its favorable pharmacokinetic parameters, the most effective treatment to remove lithium from serum is intermittent hemodialysis $[12,13]$. The prognosis of patients with LI varies, ranging from full recovery to long-lasting neurologic deficits. The most concerning long-term deficit or dysfunction of lithium toxicity is the syndrome of irreversible lithium-effectuated neurotoxicity (SILENT). SILENT is characterized by a broad spectrum of neuropsychiatric symptoms that follows lithium toxicity and may persist long after serum lithium normalization. Clinical features may include brainstem and/or cerebellar dysfunction, extrapyramidal features, and cognitive impairment [14]. Prognosis is worst when symptoms persist for a longer period. Recent data suggest a low mortality (less than 1\%) [3].

\section{CASE PRESENTATION}

The patient was a 52-year-old woman admitted to the Neurology Unit for severe agitation and mental confusion. She had a history of schizoaffective disorder and was on chronic treatment with lithium carbonate $300 \mathrm{mg}$ bid, olanzapine $10 \mathrm{mg} \mathrm{qd}$, and clonazepam $0,5 \mathrm{mg}$ qd during the last 2 years. Moreover, she had a history of hypertension and dyslipidemia. Hyperparathy- 
roidism and hypercalcemia were recently diagnosed. A parathyroid adenoma was also reported. Five days before neurological symptoms onset she had an episode of fever and diarrhea. Neurological examination showed agitation, confusion, and disorientation. She also presented akathisia and diffuse tremor. At admission, laboratory investigations showed high serum lithium, hypercalcemia, high serum parathormone, mildly elevated serum creatinine, while blood count, $\mathrm{Na}^{+}$, calcitonin, hepatic, and thyroid values were normal (Table I). Electroencephalogram (EEG) showed background activity disorganization with periodic bursts of diffuse sharp waves. Brain magnetic resonance imaging (MRI) and MR-angiography were normal. Cerebrospinal fluid analysis including virologic DNA detection, bacterial antigens, and cultures were unremarkable. Screening for autoimmune encephalitis was negative. Lithium was discontinued and in the next 3 days serum lithium and serum calcium levels decreased within the normal range (Table I), but the patient showed a progressive consciousness impairment evolving in coma state. During the next 4 days, despite a high intake of glucose $5 \%$ solutions and free water by naso-gastric tube, laboratory tests showed a progressive rise of $\mathrm{Na}^{+}$up to 184 $\mathrm{mmol} / \mathrm{L}$ and an acute renal failure with increased serum creatinine and blood urea nitrogen (Table I). Urine specific weight was low. Ultrasound showed slight reduced kidney dimension and parenchyma hyperechogenicity. Diagnosis of nephrogenic diabetes insipidus (NDI) was made and hemodialysis was started. After the first treatment, $\mathrm{Na}^{+}$ serum level decreased to $164 \mathrm{mmol} / \mathrm{L}$ without clinical improvement and patient died the day after for cardiac arrest.

\section{DISCUSSION}

Lithium is the first-line therapy for bipolar disorder and is also widely used in refractory depression and in cluster headache prevention. Lithium has a narrow therapeutic index and toxicity is frequent in patients taking this agent $[1,2,4,5]$. Longterm lithium use may lead to episodes of intoxication, that represent a considerable risk for long-term morbidity and even mortality. In our patient, who was on long-

\begin{tabular}{|l|c|c|c|c|}
\hline \multicolumn{1}{|c}{ Parameter } & \multicolumn{2}{c}{$\begin{array}{c}\text { At admission } \\
\text { days after } \\
\text { admission }\end{array}$} & $\begin{array}{c}\text { 7 days after } \\
\text { admission }\end{array}$ & Normal range \\
\hline Lithium (mmo/L) & 1.3 & 0.8 & - & $0.6-1.2$ \\
Calcium (mg/dL) & 11.9 & 9.4 & - & $8.6-10.2$ \\
Creatinine (mg/dL) & 1.33 & 1.97 & 2.49 & $0.57-1.11$ \\
BUN (mg/dL) & 45 & 89 & 145 & $10-50$ \\
Sodium (mmol/L) & 138 & 173 & 184 & $136-145$ \\
PTH (pg/mL) & 427 & - & - & $12-75$ \\
Calcitonin (pg/mL) & 4 & - & - & $0-10$ \\
USG (n) & - & 1004 & - & $1005-1035$ \\
Potassium (mmol/L) & - & - & 4.5 & $3.5-5.1$ \\
\hline
\end{tabular}

What should the clinician ask him/herself or the patient?

- Is the patient in therapy with neuroleptics, selective serotonin reuptake inbibitors (SS$R I s)$, or amiodarone?

- Has the patient a history of thyroid or parathyroid dysfunction?

- Has the patient a history of renal failure?

- Does the patient report a recent condition of dehydration, such as fever or diarrhea?

- Are the clinical features attributable to central nervous system lithium intoxication, hypercalcemia, or hypernatremia?

- Is hemodialysis indicated for lithium intoxication?

- When should hemodialysis be started?

Table I. Laboratory findings at admission, 3 , and 7 days after the admission

BUN $=$ Blood Urea Nitrogen; PTH = Parathyroid hormone;

$\mathrm{USG}=$ Urine Specific Gravity 
term lithium treatment, an episode of fever and diarrhea and consequently dehydration contributed to induce intoxication. Our patient had a spectrum of severe neurological symptoms despite a very mild increased lithium serum level $(1.3 \mathrm{mmol} / \mathrm{L}$; therapeutic range $0.6-1.2)$, as already reported in other studies $[1,3]$. This may be partially explained by the lithium characteristics: it is one of the lightest elements of the periodic table, is easily distributed throughout total body water, and does not bind serum proteins. Lithium is rapidly absorbed by kidneys, thyroid, and bones and lately by the brain. In case of acute and acute-onchronic intoxication, only lithium serum concentrations determined at least 6 hours after ingestion allow adequate interpretation of intoxication severity. In long-term lithium therapy, serum concentration may not reflect tissue levels, resulting in a poor correlation between lithium level and toxicity as in our patient. Indeed, intoxication can occur even despite normal lithium lev$\mathrm{el}$, as it was described in the literature [3]. Moreover, in our patient symptoms such as tremor, confusion, consciousness impairment may be worsened by hypercalcemia, which can overlap neurologic LI symptoms. In our patient, hypercalcemia was associated with hyperparathyroidism. Parathyroid involvement is a rare adverse effect of lithium therapy $[4,5,9]$ and was already ongoing in this patient. Lithium was promptly discontinued and hypercalcemia corrected, but the patient did not improve. Three days after, she developed acute renal failure and NDI, another rare effect of $\mathrm{LI}[6,7]$. This condition lead to severe hypernatremia despite a high intake of fluids. Hemodialysis was started, but, after the first treatment, the patient died for cardiac arrest. Autoptic examination was not performed, but prob-

\section{Keypoints}

- In lithium intoxication, neurological symptoms are common and may occur when the dosage is close to the therapeutic range or even in the normal therapeutic range

- Less frequently, lithium therapy is associated with hyperparathyroidism and hypercalcemia, which can overlap neurotoxic symptoms

- Nephrogenic diabetes insipidus is a rare adverse effect of lithium intoxication, which can lead to a life-threatening hypernatremia

- Hemodialysis should be promptly started in case of severe adverse effects or poor clinical outcome, independently of lithium serum level ably the patient died due to the neurotoxicity of severe and prolonged hypernatremia. Generally, treatment guidelines for lithium intoxication vary depending on the degree of toxicity. In case of mild toxicity, lithium discontinuation may be sufficient. In moderate intoxication, fluid infusion with saline solutions are recommended along with gastric lavage and bowel irrigation to enhance lithium elimination. In the most severe cases, defined by lithium serum levels $>4.0$ $\mathrm{mmol} / \mathrm{L}$ or severe clinical symptoms, especially consciousness impairment, hemodialysis should be performed. When hemodialysis is required, it is usually repeatedly performed to avoid lithium rebound with a paradoxical deterioration in consciousness, caused by a redistribution of lithium from tissues to the plasma. Serum lithium levels and renal function should be monitored, so that the treatment can be adjusted as necessary [13].In our case, hypercalcemia features overlapping with neurological symptoms of LI and the renal failure associated with NDI causing a severe and refractory hypernatremia lead the patient to death. Despite overlapping symptoms, probably NDI and the associated severe and non-responsive hypernatremia were responsible for the clinical worsening.

\section{CONCLUSIONS}

In patient with LI, clinicians should recognize common neurotoxic symptoms, but also rare and life-threatening adverse effects such as hypercalcemia and NDI. The serum lithium concentrations should be only a guide to avoid toxicity and should always be considered in the context of patient history and clinical findings. Therefore, any patient suspected of LI requires immediate and appropriate care. In most cases, renal failure, hypernatremia, and hypercalcemia can be safely managed without needing intensive care. Hemodialysis is indicated for lithium serum level $>4 \mathrm{mmol} / \mathrm{L}$, but, in case of severe adverse effects or poor clinical outcome, it should be promptly performed regardless of lithium serum level. In conclusion, recommendations for the safe use of lithium include correct patient selection and periodic laboratory and clinical monitoring. This may contribute to prevent the potentially harmful intoxication of this effective and widespread medication. 


\section{Consent to publication}

The consent to publication was obtained from a relative of the patient here described.

Funding

No funding has been obtained for this article.

\section{Conflicts of Interests}

The authors declare that they have no conflicts of interests concerning the topics of this article.

\section{REFERENCES}

1. Oruch M, Elderbi MA, Khattab HA, et al. Lithium: a review of pharmacology, clinical uses, and toxicity. Eur J Pharmacol 2014; 740: 464-73; https://doi.org/10.1016/j.ejphar.2014.06.042

2. Ott M, Stegmayr B, Salander Renberg E, et al. Lithium intoxication: Incidence, clinical course and renal function - a population-based retrospective cohort study.JPsychopharmacol 2016; 30: 1008-19; https://doi.org/10.1177/0269881116652577

3. Baird-Gunning J, Lea-Henry T, Hoegberg LCG, et al. Lithium Poisoning. J Intensive Care Med 2017; 32: 249-63; https://doi.org/10.1177/0885066616651582

4. McKnight RF, Adida M, Budge K, et al. Lithium toxicity profile: a systematic review and metaanalysis. Lancet 2012; 379: 721-8; https://doi.org/10.1016/S0140-6736(11)61516-X

5. Shine B, McKnight RF, Leaver L, et al. Long-term effects of lithium on renal, thyroid, and parathyroid function: a retrospective analysis of laboratory data. Lancet 2015; 386: 461-8; https:// doi.org/10.1016/S0140-6736(14)61842-0

6. Erden A, Karagöz H, Başak M, et al. Lithium intoxication and nephrogenic diabetes insipidus: a case report and review of literature. Int J Gen Med 2013; 6: 535-9; https://doi.org/10.2147/ IJGM.S46383

7. Ott M, Forssen B, Werneke U. Lithium treatment, nephrogenic diabetes insipidus and the risk of hypernatraemia: a retrospective cohort study. Ther Adv Psychopharmacol 2019; 9: 2045125319836563; https://doi.org/10.1177/2045125319836563

8. Lazarus JH. Lithium and thyroid. Best Pract Res Clin Endocrinol Metab 2009; 23: 723-33; https://doi.org/10.1016/j.beem.2009.06.002

9. Lehmann SW, Lee J. Lithium-associated hypercalcemia and hyperparathyroidism in the elderly: what do we know? J Affect Disord 2013; 146: 151-7; https://doi.org/10.1016/j.jad.2012.08.028

10. Shapiro HI, Davis KA. Hypercalcemia and "primary" hyperparathyroidism during lithium therapy. Am J Psychiatry 2015; 172: 12-5; https://doi.org/10.1176/appi.ajp.2013.13081057

11. Kayrak M, Duman C, Gul EE, et al. A bizarre electrocardiographic pattern due to chronic lithium therapy. Ann Noninvasive Electrocardiol 2010; 15: 289-92; https://doi.org/10.1111/ j.1542-474X.2010.00366.x

12. Haussmann R, Bauer M, von Bonin S, et al. Treatment of lithium intoxication: facing the need for evidence. Int J Bipolar Disord 2015; 3: 23; https://doi.org/10.1186/s40345-015-0040-2

13. Decker BS, Goldfarb DS, Dargan PI, et al. Extracorporeal Treatment for Lithium Poisoning: Systematic Review and Recommendations from the EXTRIPWorkgroup. Clin J Am Soc Nephrol 2015; 10: 875-87; https://doi.org/10.2215/CJN.10021014

14. Adityanjee, Munshi KR, Thampy A. The syndrome of irreversible lithium-effectuated neurotoxicity. Clin Neuropharmacol 2005; 28: 38-49; https://doi.org/10.1097/01. wnf.0000150871.52253.b7 